\title{
Research Mechanical Test of Accelerated Aging Method for Carbon Fiber Reinforced Polymer Composites
}

\author{
Liu Xu \\ Naval Aeronautical Engineering Academy Qingdao \\ Campus \\ Qingdao, 266041, People's Republic of China \\ liuxulw1@126.com \\ Wang Li \\ Naval Aeronautical Engineering Academy Qingdao \\ Campus \\ Qingdao, 266041, People's Republic of China \\ wangli@163.com
}

\author{
Chen Yueliang \\ Naval Aeronautical Engineering Academy Qingdao \\ Campus \\ Qingdao, 266041, People's Republic of China \\ liuxulwl@126.com
}

\author{
XI Liang \\ Department of Scientific Research \\ Naval Aeronautical Engineering Academy \\ Yantai, 264001, People's Republic of China \\ 3800294@163.com
}

\begin{abstract}
It is an important engineering problem that affected by the environmental factors, the performance of aircraft structure of carbon fiber reinforced polymer (CFRP) under the Marine environment has depredated; and it is an important means of analysis of CFRP material degradation by accelerating aging mechanics experiments under laboratory conditions. First of all, a laboratory accelerated aging spectrum of some sea area is compiled under the foundation of the sea temperature and humidity spectrum ultraviolet irradiation, and with the principle of damp and hot effection is equal to light endowment for CFRP sheet damage failure; Secondly, combining with this accelerated aging spectrum, the accelerated aging experiments have been done for the one-way and the single experiment conduct; Finally, the experimental results for hygroscopic rate and morphology have been analysis. It is showed that the accelerated aging 1a later, the moisture absorption rate is increasing, and the macro morphology changed obviously, the moisture absorption affection is most obvious by $0^{\circ}$, followed by $90^{\circ}$, the anti-aging performance is best by $45^{\circ}$.
\end{abstract}

Keywords-CFRP; accelerated aging experiment; Marine environment spectrum; analysis of moisture absorption rate; morphology analysis

\section{INTRODUCTION}

When the helicopter operating some mission in a certain sea area, stubborn Marine environment caused serious damage on the aircraft structure of CFRP materials, and aging speed is serious and more than the expected by contrasted of service period on the airport and offshore,. Natural aging experiment is the most real test method of researching composite material aging problem, but it is time consuming, and low efficiency which can not be ignored. This paper will carry out accelerated aging experiments for CFRP unidirectional single plate test piece under laboratory conditions by compiling CFRP laboratory accelerated aging spectrum. By the process of experiment, the quality variation of test pieces is recorded real-timely, and macro and micro photos of test pieces are shouted and analysis in stages. By analyzing the aging test results, theoretical basis of engineering application is provided for reliability analysis of this type of material.

\section{THE COMPLEMENT OF CFRP LABORATORY ACCELERATED SPECTRUM}

\section{A. Compiling principle of Acceleration spectrum}

It was pointed out that the most important environmental factors affecting the aging of CFRP in the literature $[1,2]$ were the temperature, humidity and ultraviolet radiation, respectively. The acceleration method of ultraviolet radiation spectrum is based on the total amount of ultraviolet radiation, so it is important to discuss the acceleration method of temperature and humidity spectrum. A lot of research results show that the aging effect of the composite material is more on the quality of the resin matrix. Because of the hydrolysis of the resin material in the moisture absorption, the damage of the material is caused by the material, which affects the life of the material.

And among moisture absorption and diffusion in the resin matrix, the Fick second law is satisfied:

$$
\frac{\partial c}{\partial t}=D \frac{\partial^{2} c}{\partial^{2} h}
$$

Where, $t$ is the moisture absorption time, $h$ is the composite material thickness, $c$ is the diffusion concentration, $D$ is the diffusion coefficient. The upper integral, then: $C_{t}=G\left(C_{m}-C_{0}\right)+C_{0}$.

Where, $C_{0}$ indicates the initial moisture absorption rate of composite materials, $C_{m}$ is a function of the 
equilibrium moisture absorption rate, $G$ is a function of time ${ }^{[3]}$.

$$
\begin{aligned}
G & =1-\frac{8}{\pi^{2}} \sum_{j=0}^{\infty} \frac{\exp \left[-(2 j+1)^{2} \pi\left(D t / b^{2}\right)\right]}{(2 j+1)^{2}} \\
& \approx 1-\exp \left[-0.73\left(D t / b^{2}\right)^{0.75}\right]
\end{aligned}
$$

Assuming that, when the temperature and moisture diffusion rate remain the same, the absorption affection occurs only on the up and down surface which have larger area, and the smaller side had no moisture phenomenon, then variation of relative mass by plate absorption process can be expressed as.

$$
\begin{aligned}
G & =1-\frac{8}{\pi^{2}} \sum_{j=0}^{\infty} \frac{\exp \left[-(2 j+1)^{2} \pi\left(D t / b^{2}\right)\right]}{(2 j+1)^{2}} \\
& \approx 1-\exp \left[-0.73\left(D t / b^{2}\right)^{0.75}\right]
\end{aligned}
$$

The initial default water content $C_{0}=0$, the moisture absorption rate is:

$$
C_{t}=C_{m}\left\{1-\exp \left[-0.73\left(D t / b^{2}\right)^{0.75}\right]\right\}
$$

Based on the fact that the initial value of the moisture absorption and diffusion of the composite material is linear, the diffusion coefficient is obtained by the initial value of the moisture absorption curve:

\begin{tabular}{|c|c|c|c|c|}
\hline$\phi_{(\%)}{ }^{\mathrm{T}\left({ }^{\circ} \mathrm{C}\right)}$ & $5^{2}$ & 0 & $5^{3}$ & \\
\hline 70 & 0.155 & 0.240 & 0.327 & 0.416 \\
\hline 80 & 0.215 & 0.316 & 0.416 & 0.511 \\
\hline 90 & 0.278 & 0.391 & 0.499 & 0.599 \\
\hline$>90$ & 0.341 & 0.464 & 0.578 & 0.681 \\
\hline
\end{tabular}

$$
D=\pi\left(\frac{b}{4 C_{m}}\right)^{2}\left(\frac{C_{2}-C_{1}}{\sqrt{t_{2}}-\sqrt{t_{1}}}\right)^{2}
$$

TABLE I. CONVERSION COEFFICIENTS FOR CFRP BETWEEN TEMPERATURE-HUMIDITY AND EXPERIMENT CONDITION

Experts and scholars at home and abroad have summarized a large number of moisture absorption test results; composited materials moisture diffusion rate under different hot and humid environment is drawn. And combined with CFRP hydrothermal affection experiment under different hot and humid environment (including the mechanics performance experiment), the common accelerated hydrothermal aging experience formula for engineering's gotten as followed ${ }^{[4,5] \text { : }}$

$$
K=\frac{t_{2}}{t_{1}}=\frac{e^{-C /\left(T_{2} \phi_{2}\right)}}{e^{-C /\left(T_{1} \phi_{1}\right)}}
$$

Among them, $K$ is the accelerate coefficient, $t_{1}, T_{1}$ and $\phi_{1}$ represent the actual exposure time, temperature and humidity respectively, $t_{2}, T_{2}$ and $\phi_{2}$ represent accelerated experiment time, temperature and humidity respectively; $C$ is the parameter, when $T_{2} \leq 60^{\circ} \mathrm{C}, C=46.1$.
The conversion coefficient of CFRP between different temperature and humidity and accelerated experiment condition can be obtained, shown in TABLE I

\section{B. CFRP laboratory acceleration spectrum}

After CFRP test pieces arrived in some sea area, it takes 1a per cycle with natural exposure, but the actual exposure experiment time is only half a year, the rest of the time is used to walk back and forth and recycle and measure the test pieces.

According to the conversion coefficient between different temperature and humidity and the experiment conditions combined with the sea temperature and humidity accelerated experiment spectrum, the total time can be obtained by simulating 1a in laboratory:

$$
\begin{aligned}
T_{\text {humidity }} & =\frac{1}{2} T_{\text {humidity }}, \\
& =\frac{1}{2} \times 2504.938=1252.5 \mathrm{~h}
\end{aligned}
$$

In terms of the ultraviolet radiation acceleration, UVA UV lamp imported from the PHILIPS Germany is used for laboratory accelerated experiment. After calibrated by UV irradiation meter, the radiation intensity near the lamp tube is $70 \mathrm{w} / \mathrm{m}^{2}$. To further shorten the test time, this experiment placed accelerated test pieces near the tube, which can be considered that the intensity of accelerated irradiation is $70 \mathrm{w} / \mathrm{m}^{2}$. The total simulation time of the 1a can be obtained by the ultraviolet radiation spectrum of the sea area, which can be calculated by the following formula ${ }^{[6]}$ :

$$
T_{\text {radiation }}=\frac{1}{2} T_{\text {radiation }}=\frac{1}{2} \times\left(\frac{Q^{\prime} \times 10^{6}}{70 \times 3600}\right)
$$

Where, $Q^{\prime}$ is the total amount of ultraviolet radiation in a certain sea area throughout years. Therefore, the acceleration time of the ultraviolet radiation of the 1a cycle in the natural exposure test of a sea area is $569.29 \mathrm{~h}$, and the radiation temperature is $55^{\circ} \mathrm{C}$.

In summary, the laboratory accelerated aging experiment to simulate the 1a cycle of natural aging experiment required soaking $1252.5 \mathrm{~h}$ distilled water, and irradiating $569.29 \mathrm{~h}$ by ultraviolet. Setting: A represent the immersion test $156.56 \mathrm{~h}$; B represent the ultraviolet irradiation $71.16 \mathrm{~h}$; AB cycle test method is adopted in this experiment as shown in Fig. 1.

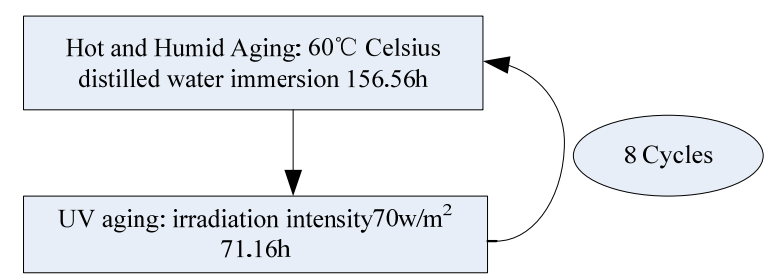

Figure 1. The experiment cycle plan 


\section{THE AGEING ACCELERATED EXPERIMENT IN LABORATORY}

\section{A. The ageing accelerated experiment}

The CFRP plates used in the ageing accelerated experiment in laboratory is single one-way shop layer board specimens designed and made on basis of domestic carbon fiber to enhance CCF materials and BA9916II resin matrix type. According to the design of the test pieces shape in GB3354-82 and GB3355-82, the laying angle is designed of $0^{\circ}, 45^{\circ}$ and $90^{\circ}$ three types as shown in Fig. 2. To prevent its own damage from fixture device while doing mechanical properties test, we specifically equip pieces of alkali EW100 without fiber pads on both sides of the test pieces. All the test pieces are made by Beijing aviation manufacturing engineering research institute.

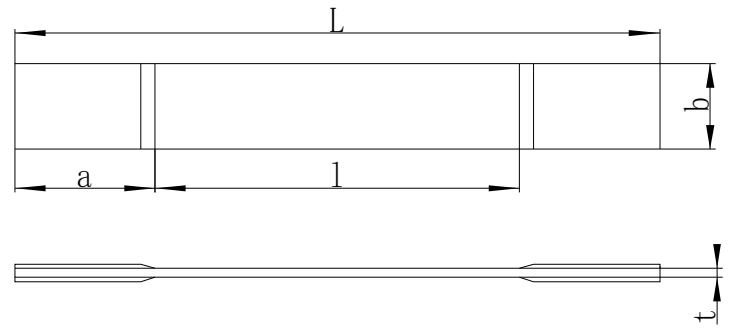

Figure 2. The design of the specimen

TABLE II. TAB.2 DIMENSION OF SPECIMEN

\begin{tabular}{|l|l|l|l|l|l|l|}
\hline \multirow{2}{*}{$\begin{array}{l}\text { Layer } \\
\text { angle }\end{array}$} & \multirow{2}{*}{$\begin{array}{l}\text { Strengthen } \\
\text { gaskets }\end{array}$} & \multicolumn{4}{|l|}{ Size } \\
\cline { 3 - 7 } & $\mathbf{L} / \mathbf{m m}$ & $\boldsymbol{b} / \mathbf{m m}$ & $\boldsymbol{t} / \mathbf{m m}$ & $\boldsymbol{a} / \mathbf{m m}$ & $\begin{array}{l}\text { Chamfering } \\
\boldsymbol{\Phi}\end{array}$ \\
\hline $0^{\circ}$ & EW100 & 230 & $12.5 \pm 0.5$ & 3 & 50 & $\geq 15^{\circ}$ \\
\hline $90^{\circ}$ & - & 170 & $25 \pm 0.5$ & 4 & 50 & - \\
\hline $45^{\circ}$ & EW100 & 250 & $25 \pm 0.5$ & 4 & 50 & $\geq 15^{\circ}$ \\
\hline
\end{tabular}

The main testing device used in this experiment includes: SHHW21.600 A II thermostatic water tank (made by Tianjin, LTD), PJZP (made by Qingdao sol test equipment co., LTD ). In the process of test, the test pieces should be placed and weighed on high precision electronic balance (made by Shanghai precision scientific instrument co., LTD ) in every 12 hours. According to the method ruled in GBT12626.8-1990, The balance should be performed under the condition of the relative humidity of $65 \pm 5 \%$ and temperature of $20 \pm 2^{\circ} \mathrm{C}$ after the test piece has constant mass so that the testing data can be read. The entire test process is about $1882 \mathrm{~h}$ long, so the surface of test pieces would be filmed microscopically at the end of each test, for observing its changing law of morphologies.

\section{$B$. The analysis of testing results}

Define the primary mass as $\mathrm{m} 0$, after a period as long as $t$, the mass of test piece is $m_{t}$. Then the rate of mass changing (it is also called the moisture absorption rate in the following) can be expressed as:

$$
r(t)=\frac{m_{t}-m_{0}}{m_{0}} \times 100 \%
$$

In the process of the first soak test, the weighing of the experiment product is recorded. Find the test pieces with the same laying angle, take the average. Then the moisture absorption rate can be acquired. The moisture curve of different laying angle in the first soak cycle is draw and shown in Fig. 3.

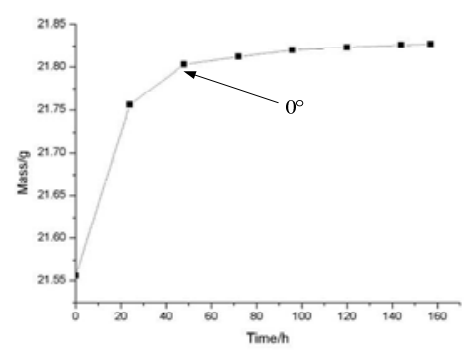

(a)

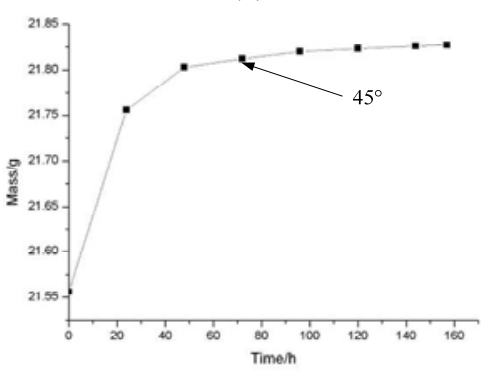

(b)

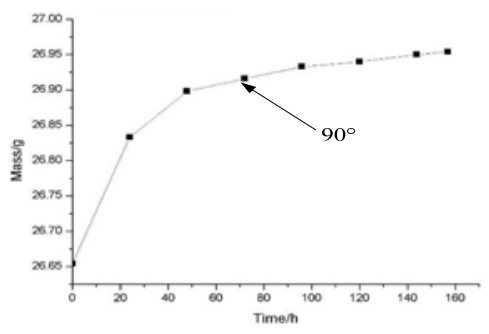

(c)

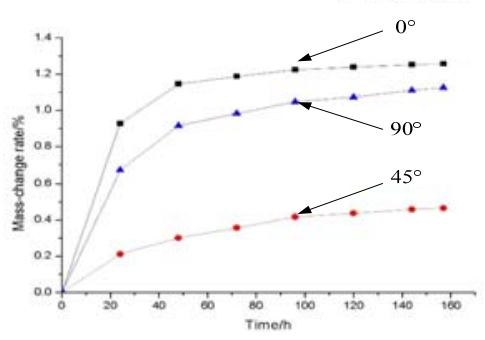

(d)

Figure 3. The mass-change for specimens when soaked during the first cycle

Comparing the different mass change curves of the test pieces of carbon fiber lying angle in the first soak cycle showing above, we can find that the test pieces' mass change curves have a similar overall trend. In the first soak cycle, $0^{\circ}$ test piece has a greatly increase on mass during the first hours of the first soak cycle, although the volume is smallest, the added mass is greatest during the first hours; the increase during the last 40 hours is tiny, similar to a straight level line. At the 
same time, the $90^{\circ}$ test piece's mass increase also slows down after the same soaking period, the mass change curve is similar to a linear function with small slope, ranking first among the three test pieces. Though it does not has as strong effect of moisture absorption as $0^{\circ}$ test piece, it may have some relation with the relative larger surface area. However, $45^{\circ}$ test piece is different. Its' volume ranks first among the three pieces, but its' mass increase is much smaller than the 0 o test piece and the $90^{\circ}$ test piece during the first 24 hours. Meanwhile, we also find that along about 157 hours test progress, the mass data nearly obeys a quadratic function. Although the mass monotone decreases after the first 24 hours, it still keeps a stable increase by the end of soaking, and it's more obvious compared with the $0^{\circ}$ test piece and the $45^{\circ}$ test piece-increasing 0.01 gram during the last 13 hours, which is five times more than the $0^{\circ}$ test piece.

Clearly, the former two have a much higher moisture absorption rate than the latter one, the $0^{\circ}$ test piece has the strongest moisture absorption effect with the $45^{\circ}$ test piece following. The gained mass amount decreases a lot because this two quickly reaches the range of the transient saturation moisture absorption. On the contrary, the $45^{\circ}$ test piece is still far from the range of the range of the transient saturation moisture absorption, so it keeps a obvious increase.

During the test, the test pieces should be filmed macroscopically and microscopically regularly, observing the changing law of morphologies of single layer board surface caused by aging. The CFRP plates has not been damaged and surface resin is also in a good state at the beginning of the test, so it's almost the same macroscopically and microscopically, and the orientation of carbon fiber could not be found. In the first two aging cycle tests, composite materials surface morphologies just change a little, shown in Fig. 4.
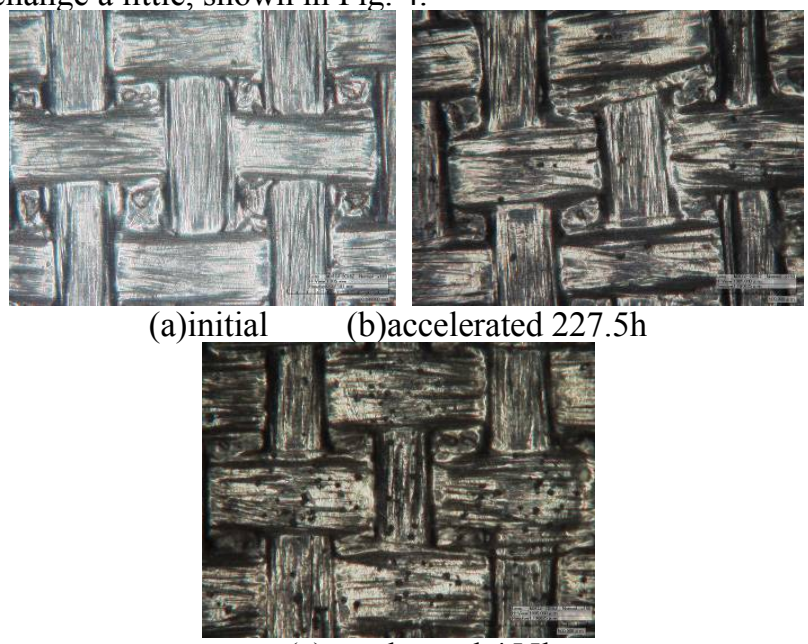

(c)accelerated $455 \mathrm{~h}$

Figure 4. Microscope photos of specimen for accelerated test during the first 2 cycles $(\times 160)$

Besides the test, we took photos of the panel both in macroscopic view and also in microcosmic, to find the changing laws of the pattern because of the age. In the prime test, because the CTRP pattern was not damaged, so the macroscopic photo and microcosmic photo were showing no differences. It's hard to find the carbon fiber arrange direction.

In the process of initial state, aging speed up $911 \mathrm{~h}$,to speed up the test at the end of the macro morphology photos. In the test before the start, CFRP board is black, in trials for four after cycle, the surface of the material color began to be turned into a pale black. Fiber soaked in direction when paper more clear. In speed up at the end of the test, the surface of the resin ash to black, the direction of the fiber arrangement has more clear. The surface of the resin consumption of serious damage. These phenomena in microscopic photo more intuitive in Fig. 5.

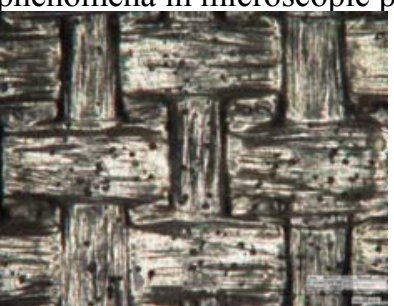

(a)Accelerated $455 \mathrm{~h}$

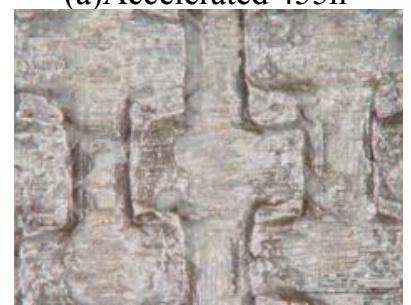

(c)Accelerated $1366 \mathrm{~h}$

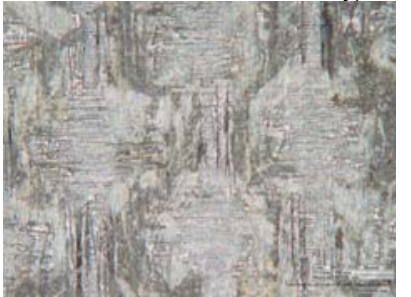

(b)Accelerated 911h

(d)Accelerated $1822 \mathrm{~h}$

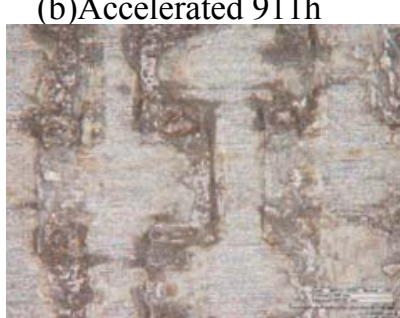

Figure 5. Microscope photographs of $\operatorname{specimen}\left(0^{\circ}\right)$ during accelerated test $(\times 160)$

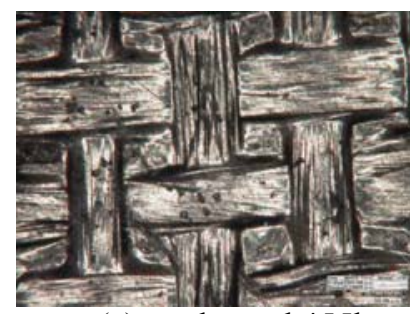

(a) accelerated $455 \mathrm{~h}$

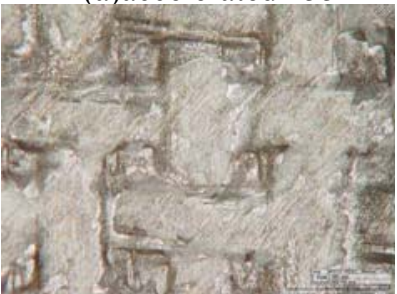

(c)accelerated $1366 \mathrm{~h}$

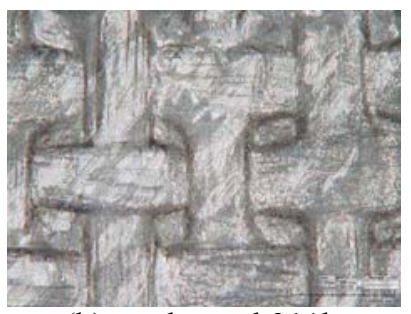

(b)accelerated $911 \mathrm{~h}$

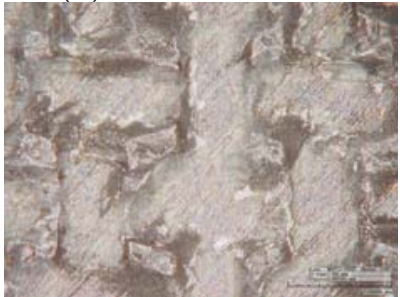

(d)accelerated $1822 \mathrm{~h}$
Figure 6. Microscope photographs of $\operatorname{specimen}\left(45^{\circ}\right)$ during accelerated test $(\times 160)$

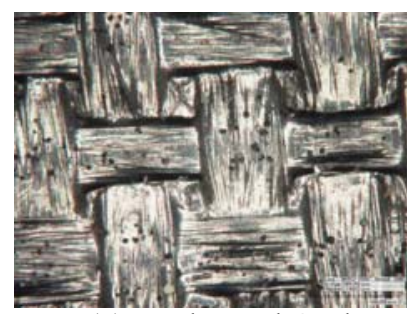

(a)accelerated $455 \mathrm{~h}$

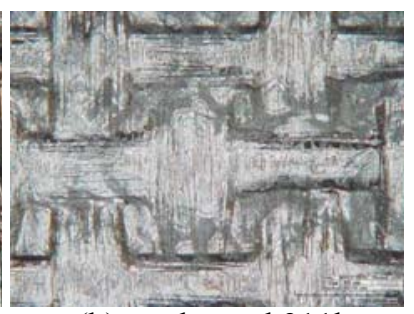

(b)accelerated $911 \mathrm{~h}$ 

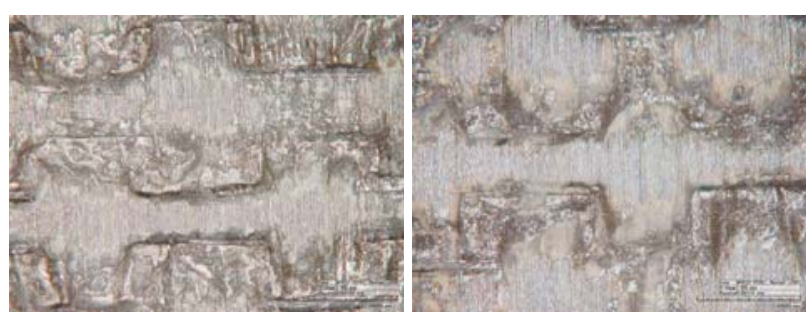

(c)accelerated $1366 \mathrm{~h}$

(d)accelerated $1822 \mathrm{~h}$

Figure 7. Microscope photographs of specimen $\left(90^{\circ}\right)$ during accelerated test $(\times 160)$

It is not hard to see that, after the accelerated aging test carries out 911 hours, namely, 4 cycles later, the surface of resin matrix has turned into the small flake off, from some black spot destruction, but the affection of resin package remains relatively intact, and it can be observed in the local scope that small amount of fiber bundle is exposed, and laying angle is visible. After accelerated aging $1366 \mathrm{~h}$, fiber bare area is further expanded, the resin layer is missing lightly only within the scope of the local concentration, and a considerable amount of matrix has "crocodile" phenomenon. After the accelerated test, resin layer lost more seriously, carbon fiber laying angle is clearly visible, the area covered by a resin layer is further reduced.

By contrast of Fig. 5(d) , Fig. 6(d) and Fig. 7(d), we can find that during the same period of aging monolayer plate specimen in different laying angle, $0^{\circ}$ and $90^{\circ}$ specimen is much more serious. In the late test, the resin layer peeling phenomenon in great quantities appeared in the two groups of specimens, and the whole row of fiber is bare in the vertical angle direction of fiber laying, and surface microstructure is more smooth. And $45^{\circ}$ specimen is not so: the resin layer covering affection is still relatively well until the end of the test, and strip shape resin covers along the fiber direction except the thick resin layer, which showed stronger anti-aging ability obviously. This phenomenon matches the results of section 3.2.2.1 which showed that specimen hygroscopic rateis different from different test pieces: the faster specimens aging rate is, the more serious moisture absorption rate of resin matrix damage.

\section{CONCLUSION}

This paper carried out accelerated aging experiments for CFRP unidirectional single plate test piece by compiling CFRP laboratory accelerated aging spectrum.
The experimental results for hygroscopic rate and morphology have been analysis. It is showed that the accelerated aging 1a later, the moisture absorption rate is increasing, and the macro morphology changed obviously, the moisture absorption affection is most obvious by $0^{\circ}$, followed by $90^{\circ}$, the anti-aging performance is best by $45^{\circ}$.

\section{REFERENCES}

[1] Cao Chunan, Wang Guangwei, Li Xinglian. Natural environmental corrosion of Chinese materials[M] Beijing :Chemical Industry Press 2005, pp.163-164

[2] Chen Yueliang, Jin Ping, Lin Dianxiong. Naval aircraft structure corrosion control and strength evaluation[M], Beijing :National Defence Industry Press. 2009, pp.14-15

[3] C.H.Shen,G.S,Spring, moisture absorption and de-sorption of composite materials, Journal of Composite Materials, 1976, pp. 51

[4] Liu wenyan, He Xiaofan, Corrosion / aging control and prolonging the calendar life of aircraft structure technology[M],Beijing : National Defence Industry Press. 2010, pp. 203-204

[5] Liu Chengchen, Wang Haowei, Yang xiaohua. Study on accelerated environmental spectra of different materials in marine atmospheric environment $[\mathrm{J}]$. Equipment environment engineering.2013, pp.18-23

[6] Wang Dongdong. Study on aging of carbon fiber / epoxy composite materials in the South China Sea[D]. Naval Aeronautical Engineering Institute.

[7] Ray.B C.Temperature effect during humid aging on interfaces of glass and carbon fibers reinforced epoxy composites[J].Journal of Colloid and interface Science,2006, pp.111-117

[8] Cai. H, Miyano. Y and Nakada. M . Long-term open-hole compression strength of CFRP laminates based on strain invariant failure theory $[\mathrm{J}]$. Journal of Thermoplastic Composite Materials, 2009, pp.63-81

[9] Miyano. Yasushi, Hongneng. Formulation of long-term creep and fatigue strengths of polymer composites based on accelerated testing methodology[J]. Journal of Composite Materials, 2008, pp.1897-1919

[10] Li Rongbao. Moisture absorption and hygrothermal aging in abismaleimide resin and its carbon fiber composites[D]. Macromolecular Science and Engineering in The University of Michigan, USA, 2001, pp.225-234

[11] Cervenka A J, Bannister D J, Young R J, Moisture absorption and interfacial failure in aramid/epoxy composites $[\mathrm{J}]$. Composites PartA, 1998, pp.1137-1144 